\title{
Should heart, lung, and liver transplant recipients receive immunosuppression induction for kidney transplantation?
}

\begin{abstract}
Ranney DN, Englesbe MJ, Muhammad W, Al-Holou SN, Park JM, Pelletier SJ, Punch JD, Lynch RJ. Should heart, lung, and liver transplant recipients receive immunosuppression induction for kidney transplantation? Clin Transplant 2010: 24: 67-72. (C) 2009 John Wiley \& Sons A/S.

Abstract: As the outcomes of heart, liver, and lung transplantation continue to improve, more patients will present for subsequent renal transplantation. It remains unclear whether these patients benefit from induction immunosuppression. We retrospectively reviewed induction on solid organ graft recipients who underwent renal transplant at our center from January 1, 1995 to March 30, 2007. Induction and the non-induction groups were compared by univariate and Kaplan-Meier analyses. There were 21 patients in each group, with mean follow-up of 4.5-6.0 years. Forty-seven percent of patients receiving induction had a severe post-operative infection, compared with $28.6 \%$ in the non-induction group $(\mathrm{p}=\mathrm{NS})$. The one yr rejection rate in the induction group was $9.5 \%$ compared with $14.3 \%$ for non-induction $(\mathrm{p}=\mathrm{NS})$. One-yr graft survival was $81.0 \%$ and $95.2 \%$ in the induction and non-induction group ( $\mathrm{p}=\mathrm{NS}$ ). In summary, there is a trend toward lower patient and graft survival among patients undergoing induction. These trends could relate to selection bias in the decision to prescribe induction immunosuppression, but further study is needed to better define the risks and benefits of antibody-induction regimens in this population.
\end{abstract}

\author{
D.N. Ranney ${ }^{a}$, M.J. Englesbe ${ }^{b}$, \\ W. Muhammad ${ }^{a}$, S.N. Al-Holou ${ }^{a}$, \\ J.M. Park', S.J. Pelletiera, \\ J.D. Punch ${ }^{\mathrm{a}}$ and R.J. Lynch ${ }^{\mathrm{a}}$ \\ aDepartment of Surgery, Division of \\ Transplantation, "'Michigan Surgical \\ Collaborative for Outcomes Research and \\ Evaluation, ${ }^{\circ}$ College of Pharmacy and \\ Department of Pharmacy Services, University of \\ Michigan, Ann Arbor, MI, USA
}

\begin{abstract}
Key words: induction - renal transplant simulect sensitization - thymoglobulin transplant survival
\end{abstract}

Corresponding author: Michael J. Englesbe, MD, University of Michigan Health System, 2926 Taubman Center, 1500 E. Medical Center Drive, Ann Arbor, Ml 48109-0331, USA. Tel.: (734) 936-8363; fax: (734) 763-3187; e-mail: englesbe@med.umich.edu

Accepted for publication 30 December 2008
As the outcomes of solid organ transplantation continue to improve, more heart, lung, and liver transplant recipients will require transplantation for end-stage renal failure. Progression to chronic renal failure in patients with a non-renal transplant is a morbid outcome, and is associated with a fourfold increased risk of death (1). Renal transplantation is increasingly being offered to these patients as a means of renal replacement therapy (2). Unfortunately, even large multi-organ transplant centers have limited experience with these complex patients, and to date, only smaller series are available in the literature to guide decisions in management (2-4).

Our group has struggled with decisions regarding induction immunosuppression at the time of renal transplant in these recipients of non-renal transplants. Nationally, induction immunosuppression is being increasingly used at the time of renal transplant to reduce the incidence of rejection and improve short- and long-term outcomes (5). The potential complications of induction immunosuppression are well described, including infection and malignancy. Recipients of previous non-renal transplants are commonly considered immunologically "high risk" with a high panel reactive antibody (PRA), and conceptually might derive significant benefit from induction immunosuppression to reduce rejection and improve long-term graft function (5-9). Conversely, these same nonrenal transplant recipients are more likely to be significantly immunosuppressed prior to renal transplantation. This fact, together with possible residual physiologic deficits related to their primary disease, may render these patients more likely to be chronically ill and at increased risk for severe infectious complications directly related to induction immunosuppression.

We have anecdotally noted some recent poor outcomes following induction therapy in cardiac 
transplant recipients. To better inform our center policies, we describe our experience with induction therapy in a group of non-renal transplant recipients who receive a kidney transplant. Our hypothesis is that the infectious complications in these patients are severe and that induction should be avoided in non-renal transplant recipients at the time of renal transplantation.

\section{Methods}

Patients

Data collection and analysis was approved by the University of Michigan Institutional Review Board. All data were collected retrospectively from the internal University of Michigan electronic medical record. All adult patients who previously had received a liver, lung, or heart transplant and subsequently received a kidney transplant between January 1, 1995 and March 30, 2007 were included in the study group. Patients with simultaneous kidney and liver or heart transplants were excluded from the study. In addition, patients with insufficient data regarding whether or not they received immunosuppression induction therapy at the time of their kidney transplant were excluded from this analysis $(n=4)$. There were two patients who received a kidney following previous pancreas transplant. No follow-up data were available on one patient, and the other received only a single dose of Atgam because of the possible anaphylactic response. Accordingly, no kidney-after-pancreas patients were available for inclusion in the study.

Induction therapy was considered to be present in those who were received thymoglobulin (rabbit anti-thymocyte globulin), Atgam (equine antithymocyte globulin), or Simulect (basiliximab) at the time of kidney transplantation. Thymoglobulin was dosed intra-operatively at $3 \mathrm{mg} / \mathrm{kg}$, and again at $2 \mathrm{mg} / \mathrm{kg}$ on post-operative day 2. Post-operative doses were decreased by $50 \%$ in three patients with white blood cell counts of 1500-3000 cells/microliter, but all patients complete full courses. Basiliximab induction consisted of two $20 \mathrm{mg}$ doses. Atgam therapy was composed of $20 \mathrm{mg}$ infusions daily for 10 days. Indications for induction therapy were African American race, documented PRA $>30 \%$, minimal immunosuppression following initial transplant, or receipt of a living unrelated donor organ, with final decisions on inclusion made by the operative surgeon. All patients enrolled in induction therapy completed their prescribed course, with dose adjustments for leukopenia as necessary in the thymoglobulin group.
Maintenance therapy was comprised of a calcineurin inhibitor, mycophenylate mofetil, and a prednisone taper. Cyclosporine was used in the majority of non-induction patients $(17 / 21,81 \%)$, with one patient receiving sirolimus, and a total of four receiving tacrolimus (one crossover for intolerance of cyclosporine). In the induction group, cyclosporine was also the most common calcineurin inhibitor $(13 / 21,62 \%)$. Two patients received sirolimus, and a total of nine $(43 \%$, with three crossovers from cyclosporine) received tacrolimus.

For subsequent analysis, the exposure variable was receipt of antibody induction immunosuppression at the time of renal transplantation. Comparisons between the two groups included: clinical characteristics, maintenance immunosuppression regimen, readmission data, the incidence of infections, rejection, and cancer, as well as patient and graft survival. Readmission data included only those readmissions that occurred at the University of Michigan. All patients with biopsy-proven rejection (Banff $2 \mathrm{~A}$ of greater) were readmitted for initiation of anti-rejection therapy.

\section{Statistical analysis}

The association of donor, recipient, and transplant characteristics between the induction and the noninduction groups were compared by univariate analysis. Categorical variables were analyzed using chi-square analysis. Continuous variables were assessed with a two-tailed Student's $t$-test. Unadjusted rates of graft failure and patient survival were calculated by the method of Kaplan and Meier (10). The data were analyzed using SPSS software (version 15.0, SPSS Inc., Chicago, IL, USA).

\section{Results}

A total of 42 patients fulfilled inclusion criteria for this analysis. The reason for development of endstage renal disease was either wholly or partly ascribed to calcineurin inhibitor toxicity in 22 of the 42 patients, with causes for renal failure in the remainder including diabetic nephropathy (8), hypertension (1), and acute tubular necrosis (4), Twenty-one of these kidney transplant recipients received induction at the time of transplant, and the remaining 21 patients did not receive induction. The patient characteristics of these two study groups are detailed in Table 1. The majority of the primary transplants were for end-stage liver disease $-67 \%$ for the induction group and $71 \%$ for the non-induction group. There was a significantly 
Induction of previous transplant recipients

Table 1. Comparison of the clinical characteristics between two groups of liver, heart, and lung transplant patients who received either induction therapy or did not at the time of kidney transplantation

\begin{tabular}{|c|c|c|c|}
\hline & Induction ( $\mathrm{n}=21$ ) & Non-induction $(n=21)$ & p-values \\
\hline $\begin{array}{l}\text { Age at kidney } \\
\text { transplant }\end{array}$ & $43.0 \pm 14.5$ & $46.6 \pm 11.2$ & 0.36 \\
\hline Sex & $66.7 \%$ male (14) & $71.4 \%$ male (15) & 0.74 \\
\hline Race & 90.5\% non-black (19) & $100 \%$ non-black (21) & 0.15 \\
\hline Diabetic & $38.1 \%(8)$ & $52.4 \%(11)$ & 0.35 \\
\hline \multicolumn{4}{|l|}{ Previous transplant } \\
\hline Heart & $7(33.3 \%)$ & $4(19.0 \%)$ & \multirow[t]{3}{*}{0.24} \\
\hline Lung & 0 & $2(9.5 \%)$ & \\
\hline Liver & $14(66.7 \%)$ & $15(71.4 \%)$ & \\
\hline $\begin{array}{l}\text { Years from primary } \\
\text { transplant }\end{array}$ & $7.4 \pm 5.5$ & $8.2 \pm 5.8$ & 0.65 \\
\hline $\begin{array}{l}\text { Mean follow-up } \\
\text { since kidney transplant }\end{array}$ & $4.5 \pm 4.2$ & $6.0 \pm 3.8$ & 0.26 \\
\hline \multicolumn{4}{|l|}{ Transplant type } \\
\hline Living donor & $4(19.1 \%)$ & $14(66.7 \%)$ & \multirow[t]{2}{*}{$0.001^{*}$} \\
\hline Deceased donor & $17(80.9 \%)$ & $4(33.3 \%)$ & \\
\hline Mean PRA & $36 \pm 39$ & $21 \pm 13$ & 0.09 \\
\hline
\end{tabular}

higher proportion of deceased donor kidney transplants in the induction group. The 21 patients that received induction immunosuppression at the time of the kidney transplant were dosed with either anti-thymocyte globulin (thymoglobulin, 67\%, $\mathrm{n}=14$ ), lymphocyte immune globulin (Atgam, $24 \%, \mathrm{n}=5$ ), or basiliximab (Simulect, $10 \%$, $\mathrm{n}=2$ ). The panel reactive antibody scores at time of transplant in the induction group were heterogeneous, with PRA $<10$ in $28.5 \%$, PRA $10-40$ in $28.5 \%$, and PRA $>40$ in the remaining $43 \%$.

In general, a higher percentage of patients that received induction immunosuppression at the time of the kidney transplant were readmitted within the first year following a kidney transplant, but no comparisons reached statistical significance. One more patient was readmitted for treatment of rejection in the non-induction group than in the induction recipients $(14.3 \%$ vs. $9.5 \%, p=N S)$. A comparison of the reasons for readmissions is graphically represented in Fig. 1.

There was a tendency toward more readmissions for infection in the induction group compared with the non-induction group. A total of 23 infectious complications were noted in 16 patients. In the induction group, there were 14 infections in 10 patients, of which five were infections of the respiratory tract, one was a urinary tract infection, two were of intra-abdominal origin, three were related to surgical site infection, two others were viral gastroenteritis, and one other to CMV colitis. Six non-induction patients suffered a total of nine events, comprised of two respiratory infections, two urinary tract infections, three intra-abdominal infections, and two cases of viral gastroenteritis. Of note, no polyoma virus-related infections were documented in either group.

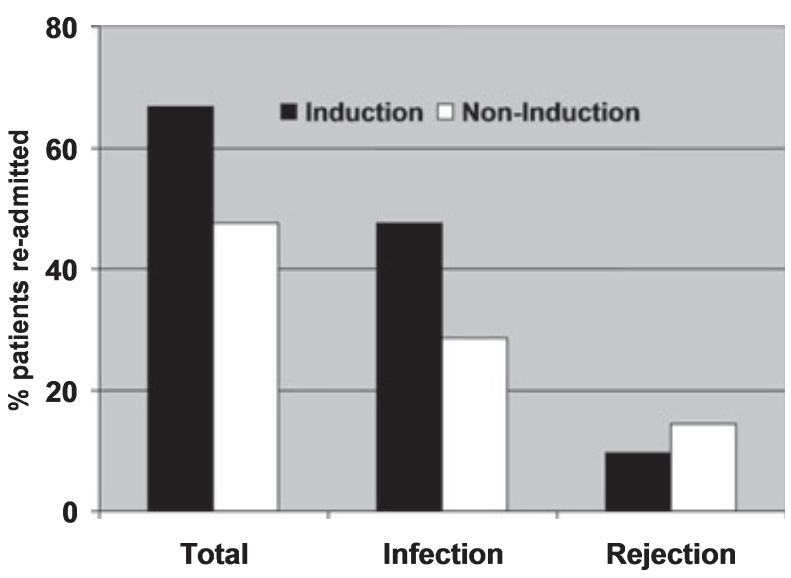

Fig. 1. The percentage of patients in the induction and in the non-induction groups who were readmitted within the first year after kidney transplant for either an infection or rejection (Banff 2A rejection or greater). $\mathrm{p}>0.05$ for all comparisons.

Patient outcomes with regard to renal transplant are summarized in Table 2. Delayed graft function was more common in the induction group $(47.6 \%$ vs. $9.5 \%, \quad \mathrm{OR}=5.0,95 \% \mathrm{CI} \quad 1.24-20.1)$. No statistically significant differences were noted in patient and graft outcomes between the induction and the non-induction groups, though there was a moderately lower rate of patient and graft survival in the induction group at one yr. Of note, there was no early graft loss in patients surviving more than one yr post-transplant. Causes for eventual graft failure listed in the "other" category of Table 2 included chronic fibrosis ( $\mathrm{n}=1$, induction group), recurrence of primary disease (one patient in each group), possible calcineurin inhibitor toxicity ( $\mathrm{n}=1$, induction group), and unknown cause (one patient, induction group). 
Table 2. Summary of the one-yr patient and graft outcomes among 42 heart, lung, and liver transplant recipients who either did or did not receive immunosuppressive induction therapy at the time of a subsequent kidney transplant

\begin{tabular}{lccl}
\hline Outcome & $\begin{array}{l}\text { Induction (\%) } \\
(\mathrm{n}=21)\end{array}$ & $\begin{array}{l}\text { Non-induction (\%) } \\
(\mathrm{n}=21)\end{array}$ & p-values \\
\hline Delayed graft function & 47.6 & 9.5 & $\mathbf{0 . 0 0 4 ^ { \star }}$ \\
One yr graft survival & 81.0 & 95.2 & 0.15 \\
One yr patient survival & 81.0 & 95.2 & 0.15 \\
Cause of death infection & $14.3(3)$ & $9.5(2)$ & 0.47 \\
Cause of death cancer & $4.8(1)$ & $4.8(1)$ & 0.47 \\
Reason for graft loss & $\mathrm{n}=8$ & $\mathrm{n}=6$ & 0.39 \\
Rejection & $12.5(1)$ & $33.3(2)$ & \\
Death & $37.5(3)$ & $50.0(3)$ & \\
Other & $50.0(4)$ & $16.7(1)$ & \\
\hline
\end{tabular}

The bold and '*' highlights value with a signficant $(<0.05) p$-value.

Primary organ (heart, lung, liver) survival was also analyzed in the two groups. Among the fortytwo patients, there was only one primary graft loss in the non-induction group, which was a recurrence of hepatitis $\mathrm{C}$ virus in a transplanted liver, ultimately leading to the patient's demise. No primary organs were lost in the induction group.

Long-term patient survival (Fig. 2) and graft survival (Fig. 3) were estimated using KaplanMeier survival analysis. In both the induction and the non-induction groups, survival declined at approximately equivalent rates. The survival rate for induced patients is consistently lower than that of non-induced patients, though this difference did not extend to a statistically significant degree. The graft survival curves demonstrate similar outcomes, and these data again were not statistically significant between induction and non-induction groups. There were four deaths within the first year

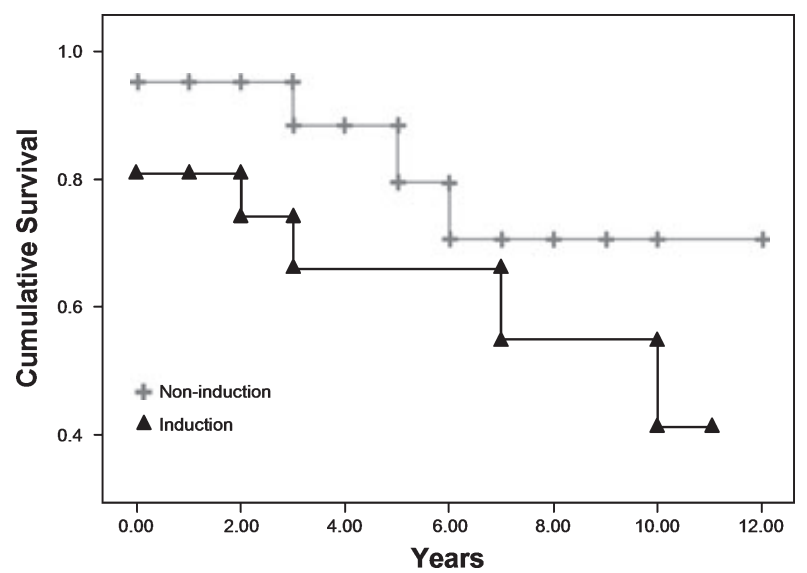

Fig. 2. Kaplan-Meier curves detailing patient survival among previous heart, lung, or liver transplant recipients who did or did not receive immunosuppressive induction at the time of kidney transplant $(\mathrm{p}=0.12)$.

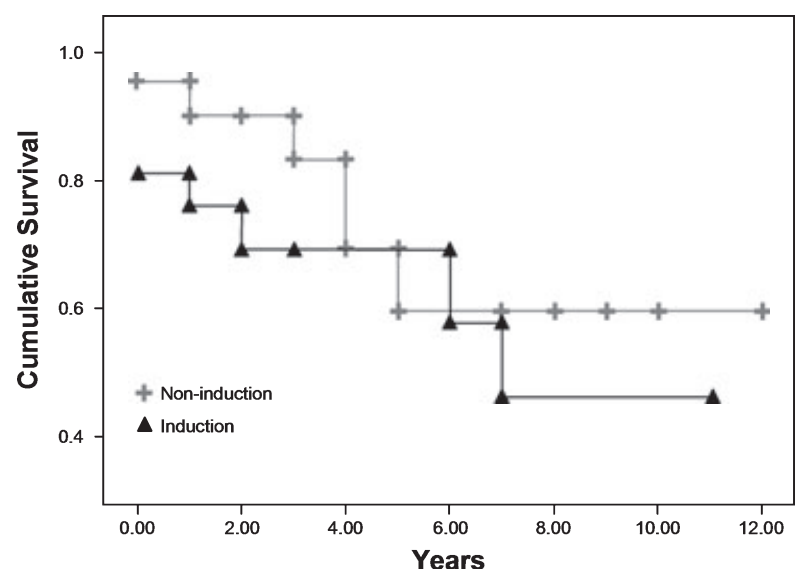

Fig. 3. Kaplan-Meier curves detailing long term kidney transplant graft survival among previous heart, lung, or liver transplant recipients who did or did not receive immunosuppressive induction at the time of kidney transplant $(\mathrm{p}=0.32)$.

in the induction group, all of whom had received thymoglobulin.

\section{Discussion}

With this study, we report on the outcomes of 42 kidney transplant patients who had previously received a non-renal solid organ transplant. Our analysis attempts to answer the question of whether these patients should receive induction immunosuppression at the time of kidney transplant. Our findings note higher delayed graft function and lower patient and graft survival in the group of patients that received induction therapy. As expected, the group of patients that received induction immunosuppression also had more infections and less rejection than the group of patients that did not receive induction. The disparity between patient and graft survival are shown in Figs. 2 and 3 is largely the result of four deaths in the first year in the induction group. Each of these patients had at least one readmission for infection, and three of the four died in the setting of sepsis. Rejection was an infrequent occurrence, and slightly less common in the induction group. The induction group had an approximately fivefold higher rate of delayed graft function than noninduction patients, but the elevated delayed graft function (DGF) risk associated with the higher proportion of deceased donors in this group is a more likely etiology of DGF than effect related to induction medications. The complexities of patient outcomes in this setting underscore the need to carefully weigh the risks and benefits of induction immunosuppression in these chronically ill transplant recipients. 
The long-term survival of liver, heart, and lung transplant patients continues to improve $(11,12)$. These patients face many potential long-term complications following solid organ transplantation, including end-stage renal disease. Specifically, chronic renal failure developed at approximately $16.5 \%$ of solid organ transferred recipients over a median follow-up time of 36 months. Our center and others are increasingly seeing non-renal solid organ transplant recipients for evaluation for kidney transplantation (2). The patient and graft survival of this select group of patients is an emerging area of study. The largest report in the literature contained 18 solid organ transplant recipients who went on to receive kidney transplants (3). In general, this study and smaller studies have reported excellent results with respect to patient and renal allograft survival inclusive of a variety of immunosuppressive strategies $(3,4)$.

There have been no previous reports specifically pertaining to the management of induction immunosuppression in patients with previous non-renal transplants who go on to require kidney transplantation. There is a significant movement in the United States toward increased utilization of induction immunosuppression at the time of kidney transplantation (5). Our center reserves induction immunosuppression for patients with a PRA $>30 \%$, African American recipients, non-immunosuppressed recipients of a prior solid organ graft, and recipients of living unrelated kidney transplants. Even though a significant number of previous nonrenal solid organ transplant recipients fulfill our center-specific criteria to receive induction immunosuppression, we have been concerned about giving such potent immunosuppression to this patient population. Several recent poor outcomes in patients who have received induction immunosuppression served as the impetus for this study.

Our series of 42 patients is the largest report of renal transplant following previous non-renal solid organ transplant, and is the first to focus on the use of induction immunosuppression in these complex patients. Even though our group has this relatively large experience, this analysis is underpowered to clearly determine whether recipients of a previous non-renal transplant should receive induction at the time of a kidney transplant. Recipients of induction therapy had less rejection, but overall had more infections, and worse survival. All four early deaths in the induction group were in patients who had received thymoglobulin. It remains unclear whether these poor outcomes are independently related to induction immunosuppression, or are related to other patient-specific factors. A higher percentage of the non-induction group received kidneys from living donors, though the reasons for this disparity are not clear. Data previously developed at our center, and were consistent with multiple reports in the literature, links living-donor organ transplant to better shortand long-term outcomes. The reasons for this advantage are likely manifold, beginning with shorter mean time on dialysis, smoother perioperative course, and better social support structure following transplantation. All of these factors have been shown to significantly impact kidney transplant outcomes $(13,14)$, and could affect our result. Certainly, the higher rate of deceased donor renal transplant in the induction group confounds analysis of the disparity in delayed graft function rates. Another potential confounding factor includes the indications for induction therapy. By protocol, recipients of induction therapy are at a higher immunologic risk for rejection. Physician discretion plays a role in this decision making, however, and could make the difference between inclusion and standard therapy. Conversely, some patients who by PRA criteria meet requirements for induction therapy were thought to be too frail, and were not given induction immunosuppression. The size of our series does not allow for a robust multivariable analysis that would control for such confounding influences. In addition, the retrospective nature of this analysis does not allow full investigation regarding the reasons patients were placed in the induction or non-induction group. A significantly larger sample size would also allow exploration of the relative superiority of one type of induction therapy in this population.

Although currently there is a relatively small number of patients receiving kidneys after previous solid organ transplants (approximately $2 \%$ of kidney transplant recipients at our center), this group of patients will undoubtedly continue to expand. When deciding upon induction immunosuppression, transplant physicians must carefully consider chronic medical conditions, degree of immunosuppression prior to kidney transplant, risk of an infectious complication, and the potential morbidity associated with acute cellular rejection. Even though our study is quite limited by both the complexity of the patients and the small sample size, induction immunosuppression does seem to be associated with poor short-term outcomes compared with the non-induction immunosuppression. Specifically, infectious complications in the induction group were more common, and overall of higher acuity, than in non-induction counterparts. Similarly, this limited analysis does not suggest any long-term benefits from induction immunosuppression. Induction immunosuppression 
should be reserved for high immunologic risk categories in previous recipients of a non-renal solid organ transplant. In the absence of large enough series to validate specific numerical cutoffs for PRA score or other criteria, decisions on induction will continue to be heavily reliant on physician discretion. As such, a multi-disciplinary group, including a practitioner who manages the patient's long-term immunosuppression prior to the kidney transplant, will be beneficial when ultimately deciding whether or not to give these complicated patients antibody induction immunosuppression.

\section{Acknowledgements}

Dr. Englesbe and Dr. Lynch are supported by a grant from the American Surgical Association Foundation and American Society of Transplant Surgeons, respectively.

\section{References}

1. Ojo AO, Held PJ, Port FK et al. Chronic renal failure after transplantation of a nonrenal organ. N Engl J Med 2003: 349: 931 .

2. Chandrakantan A, de Mattos AM, Naftel D, Crosswy A, Kirklin J, Curtis JJ. Increasing referral for renal transplant evaluation in recipients of nonrenal solidorgan transplants: a single-center experience. Clin J Am Soc Nephrol 2006: 1: 832.

3. Coopersmith CM, Brennan DC, Miller B et al. Renal transplantation following previous heart, liver, and lung transplantation: an 8-year single-center experience. Surgery 2001: 13: 457.
4. Tarnow H, Herlenius G, Friman $\mathrm{S}$ et al. Outcome of renal transplantation subsequent to liver, heart, or lung transplantation. Transplant Proc 2006: 38: 2649.

5. Meier-Kriesche HU, Li S, Gruessner RW et al. Immunosuppression: evolution in practice and trends, 1994-2004. Am J Transplant 2006: 6: 1111.

6. Ciancio G, Burke GW, Gaynor JJ et al. A randomized trial of three renal transplant induction antibodies: early comparison of tacrolimus, mycophenolate mofetil, and steroid dosing, and newer immune-monitoring. Transplantation 2005: 80: 457.

7. Hardinger KL, Schnitzler MA, Koch MJ et al. Thymoglobulin induction is safe and effective in live-donor renal transplantation: a single center experience. Transplantation 2006: 81: 1285.

8. Alangaden GJ, Thyagarajan R, Gruber SA et al. Infectious complications after kidney transplantation: current epidemiology and associated risk factors. Clin Transplant 2006: 20: 401.

9. Clesca P, Dirlando M, Park SI et al. Thymoglobulin and rate of infectious complications after transplantation. Transplant Proc 2007: 39: 463.

10. Kaplan El, Meier P. Nonparametric estimation from incomplete observations. J Am Stat Assoc 1958: 53: 457.

11. Hanto DW, Fishbein TM, Pinson CW et al. Liver and intestine transplantation: summary analysis, 1994-2003. Am J Transplant 2005: 5: 916.

12. Orens JB, Shearon TH, Freudenberger RS, Conte JV, Bhorade SM, Ardehali A. Thoracic organ transplantation in the United States, 1995-2004. Am J Transplant 2006: 6: 1188 .

13. Cohen DJ, St Martin L, Christensen Ll, Bloom RD, Sung RS. Kidney and pancreas transplantation in the United States, 1995-2004. Am J Transplant 2006: 6: 1153.

14. Danovitch GM, Cohen DJ, Weir MR et al. Current status of kidney and pancreas transplantation in the United States, 1994-2003. Am J Transplant 2005: 5: 904. 